
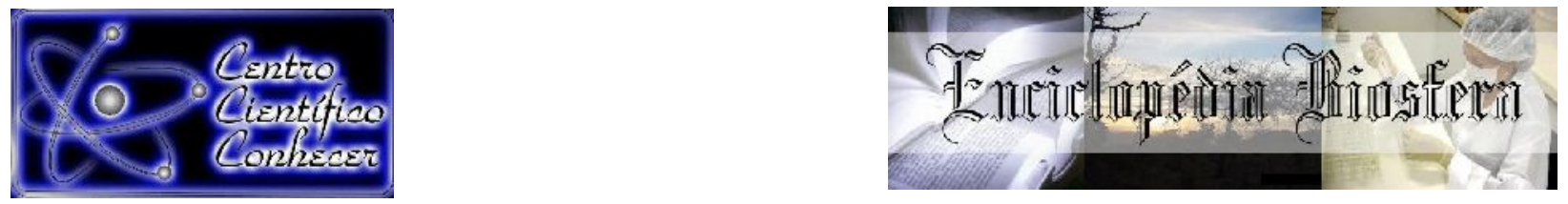

\title{
SAZONALIDADE DA COCHONILHA ESCAMA FARINHA NA CITRICULTURA DE UMA FAZENDA LOCALIZADA NA REGIÃO NOROESTE PAULISTA/SP _ ESTUDO DE CASO
}

\section{Gilson Barbara ${ }^{1}$; Dagmar Aparecida de Marco Ferro ${ }^{2}$}

${ }^{1}$ Discente do Curso de Engenharia Agronômica, Centro Universitário - UNIFUNEC. Santa Fé do Sul: SP, Tecnólogo em Produção Sucroalcooleira - UNIFUNEC, Técnico Agrícola -

Escola Técnica Agrícola/Centro Paula Souza: Jales: SP.gilsoneia@hotmail.com.

${ }^{2}$ Docente no Centro Universitário UNIFUNEC - Santa Fé do Sul: SP, Graduada em Ciências

Biológicas Universidade Federal de São Carlos: SP Mestrado em Ecologia e Recurso

Naturais: SP, Universidade Federal de São Carlos, Doutorado em Genética e Evolução: Universidade Federal de São Carlos: SP. dagmarferro@yahoo.com.br.

Recebido em: 04/10/2019 - Aprovado em: 30/11/2019 - Publicado em: 15/12/2019 DOI: 10.18677/EnciBio_2019B50

\begin{abstract}
RESUMO
As cochonilhas estão entre os insetos mais frequentemente encontrados e que causam problemas na citricultura. Os prejuízos ocorrem devido à sucção contínua da seiva e introdução de toxinas, que provocam fendas longitudinais no tronco e galhos. População elevada causa perda de vigor e produtividade da planta. Objetivo foi estudar a incidência de cochonilha escama farinha e sua dinâmica populacional na citricultura. A metodologia envolveu a identificação da praga da cultura de acordo com a inspeção realizada diariamente por folhas e troncos. Os resultados identificaram cochonilhas em todos os meses. O nível baixo populacional permitiu considerá-los como sendo uma praga secundaria.
\end{abstract}

PALAVRAS-CHAVE: Cochonilha; Citricultura, dinâmica populacional;

\section{SEASONALITY OF COCHINEAL SCALE FLOUR IN THE CITRICULTURE OF A FARM LOCATED IN THE NORTHWEST OF THE STATE OF SAO PAULO. ABSTRACT}

The cochineal are amongst the most commonly found insects and one of the most problematic of citriculture. The damage happen due to the continue suction of sap and introduction of toxins that will cause slits in trunks and stems. Elevated population causes loss in plants productivity. The goal was to study the presence of cochineal scale flour and its population dynamics in citriculture. The methodology involved the identification of the bugs in the culture according to the daily inspection of leaves and stems. The results identified cochineal presence every month. The low population level allowed them to be considered a secondary plague.

KEYWORDS: Cochineal; Population Dynamics; Citriculture

\section{INTRODUÇÃO}

As cochonilhas de carapaça estão entre os insetos mais frequentemente encontrados e que mais problemas causam aos citricultores. As consequências de não controlar este tipo de praga usualmente predispõe o citricultor a perdas significativas na quantidade e qualidade da fruta produzida e há casos em que a capacidade produtiva das plantas fica comprometida, (GARCIA et al., 2018). 
De acordo com Gravena, (2003) as plantas cítricas possuem, mundialmente, um dos maiores números de espécies de pragas agrícolas associadas, quando comparadas aos demais cultivos. Em virtude da diversidade de espécies de artrópodes-praga presentes durante todo o ciclo da cultura, alguns deles são vetores de doenças severas como é o caso dos insetos sugadores e grande parte dos custos operacionais e de produção é voltada para o controle desses organismos (YAMAMOTO; PAIVA, 2014; GARCIA et al., 2018).

Os principais insetos sugadores que ocorrem em plantas cítricas são: cochonilhas, moscas-branca, psilídeos, afídeos (Hemiptera: Sternorrhyncha), e cigarrinhas (Hemiptera: Auchenorrhyncha) (WOLFF et al., 2004; PERONTI et al., 2016). Os prejuízos ocorrem devido à sucção contínua da seiva e introdução de toxinas, que provocam fendas longitudinais no tronco e galhos, viabilizando a penetração de micro-organismos patogênicos. Além disso, reduz o vigor e pode causar a morte da planta (CORSEUIL, 1958; GRAVENA, 2001; MUHAMMAD et al., 2015).

O conhecimento da diversidade e da dinâmica populacional da cochonilha presente em citros, nas diferentes regiões do país, é fundamental para o estabelecimento de estratégias de manejo integrado de pragas, visando preservar as principais espécies de inimigos naturais presentes nos pomares. A escama farinha (Unaspis citri) é uma das principais cochonilhas de carapaça e em população elevada, provoca perda do vigor e produtividade da planta além de rachaduras no tronco que favorecem infecções por fungos e outras doenças, (MUHAMMAD et al., 2015).

A pesquisa teve por objetivo estudar a incidência de cochonilha escama farinha e sua dinâmica populacional na citricultura, bem como a influência de fatores climáticos e temperatura em uma propriedade localizada no município de Santa Fé do Sul - SP.

\section{MATERIAL E MÉTODOS}

O trabalho foi desenvolvido na propriedade Fazenda Recanto da Onça no município de Santa Fé do Sul/ SP localizada na Rodovia dos Barrageiros, km $96 \mathrm{~S} / \mathrm{N}$

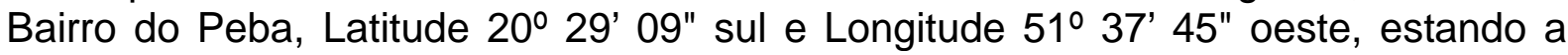
uma altitude de 370 metros. Foram analisadas $1 \%$ de 180.000 plantas da variedade Laranja Pera Rio, abrangendo um total de 131,04 hectares (FIGURA 1).

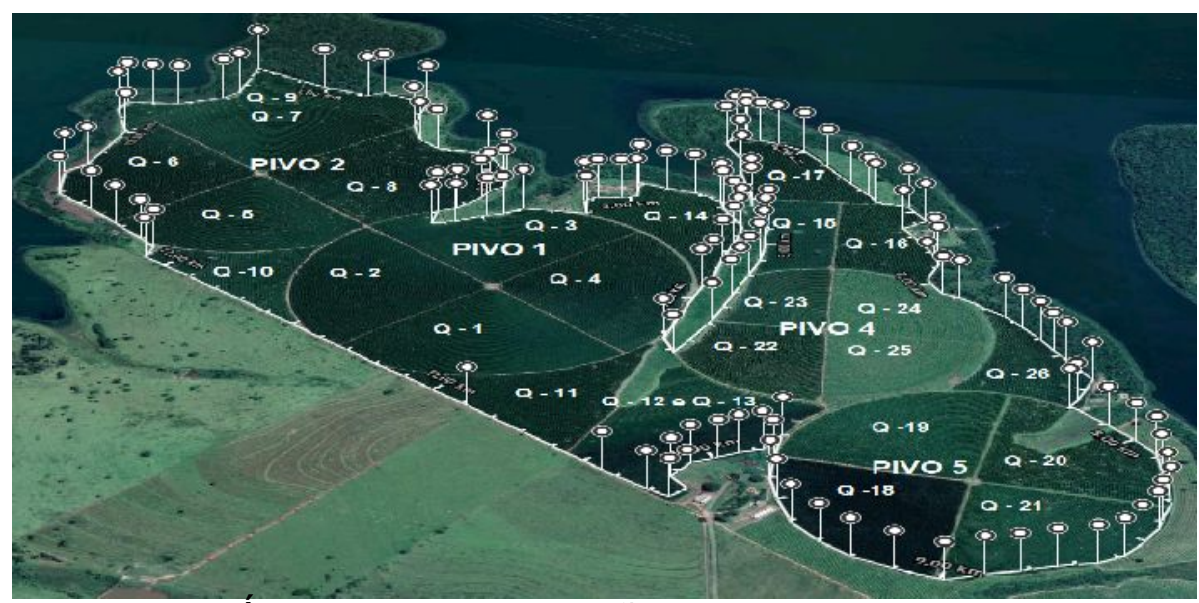

FIGURA 1: Área total de Produção Avaliada.

Fonte: GOOGLE MAPS, (2019) 
O pomar foi subdividido em quadras e a avaliação realizada no período de janeiro de 2017 a dezembro de 2018, de acordo com o cronograma de ficha de avaliação populacional de pragas e entregue ao técnico responsável pela identificação de pragas na cultura, conforme a uniformidade das plantas. $O$ inspetor técnico percorreu todas as quadras realizando o percurso em zigue-zague abrangendo assim uma área representativa. Em cada inspeção foram vistoriados pelo menos $1 \%$ das plantas do pomar avaliado. O levantamento de dados foi obtido em um período de 24 meses, com observações diárias e com retorno aos talhões no máximo a cada 15 dias, totalizando 48 repetições (levantamento de Incidências Fitossanitárias).

Foram observadas partes da planta de acordo com o comportamento de cada praga. Para a cochonilha escama farinha, as coletas das amostras foram realizadas observando sintomas de tronco e galhos, pois estes são externos (localizado na extremidade dos troncos) em todos os estádios fenológicos da planta, entre as regiões sombreadas. A cochonilha escama farinha apresenta o comportamento de se localizar na parte interna, na região do meio da copa entre os galhos, caracteriza-se por ataques severos, podendo desenvolver-se em todo o período fenológico do vegetal.

A leitura na avaliação foi manuscrita na ficha do técnico (pragueiro) de acordo com o dano provocado. Na escama farinha foi observada a presença ou ausência da praga. A presença de $10 \%$ ou mais pode ser considerado índice de dano econômico pela agressividade dos danos causados. Durante o período de avaliação foram realizadas leituras diárias das médias de temperatura máxima e mínima na propriedade, além de acompanhar diariamente o histórico pluviométrico da região local. Durante todo o período avaliado foram realizados controle com pulverização química, utilizando inseticidas e óleo mineral para o controle da cochonilha, sendo realizada quando o nível de incidência atingia números acima de $10 \%$ de plantas infestadas no pomar.

\section{RESULTADOS E DISCUSSÃO}

De acordo com os dados obtidos e observados no decorrer da pesquisa, foi possível constatar a presença de cochonilhas escama farinha em citros durante todo o ano em nível considerado baixo, embora tivessem ocorridos picos populacionais em diferentes ocasiões durante os anos avaliados conforme visualizados nos Gráficos 1 e 2.

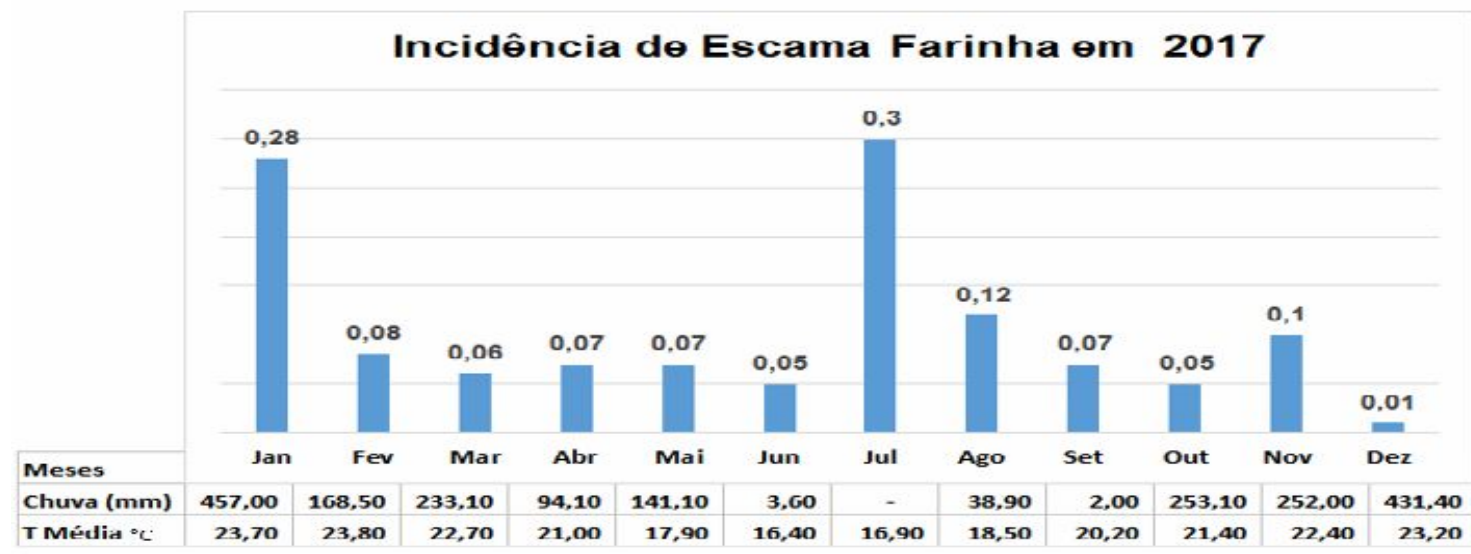

GRÁFICO 1: Incidência Fitossanitária de Escama Farinha.

FONTE: Próprios autores. 


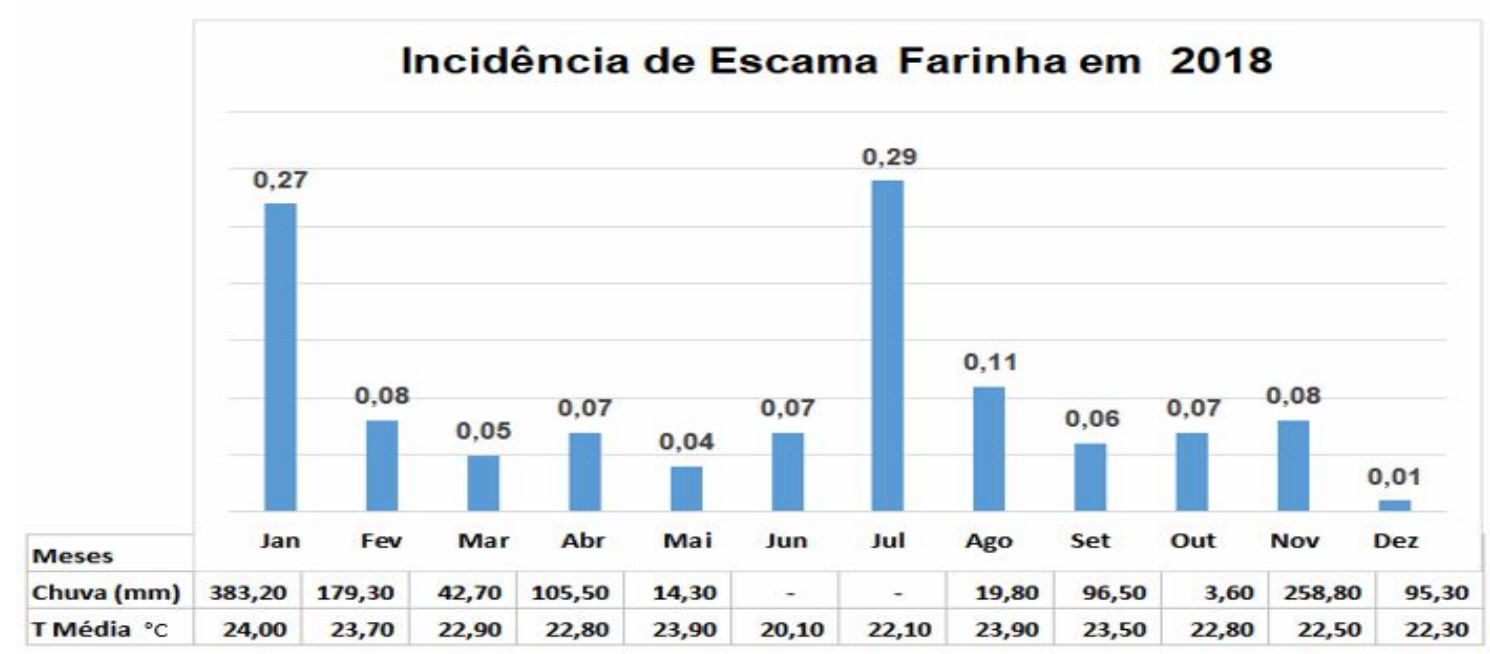

GRÁFICO 2: Incidência Fitossanitária de Escama Farinha.

FONTE: Próprios autores.

Tanto o Gráfico 1 como o Gráfico 2, mostram que a escama farinha apresentou índices fitossanitários baixos com ocorrências durante os 24 meses de avaliação. Entretanto, dois meses se destacaram, janeiro e julho de 2017 e 2018. Segundo a Fundecitrus (2016), o período de reprodução da cochonilha tem início em setembro e a incidência maior ocorre no outono e inverno, embora nas estações chuvosas sejam verificados picos populacionais elevados. Em fevereiro de 2016 foi período de identificação da presença da cochonilha em troncos e entre abril até julho de 2016 foi observada a dispersão das cochonilhas, (OLIVEIRA et al., 2016).

A cochonilha Escama Farinha é agressiva e os sintomas na planta são rachaduras no tronco e ramos (CORREIA, 1996; TAVARES, 1997; OLIVEIRA et al., 2016). A inspeção periódica do pomar é de fundamental importância, pois permite detectar a presença das pragas e o seu grau de infestação. A decisão de aplicar ou não o inseticida deve ser tomado após a inspeção e a avaliação da presença das insetos pragas e dos inimigos naturais. O Manejo Integrado de Pragas - MIP é uma técnica que mantém o número de pragas sempre abaixo do nível em que causam danos para o ambiente. O controle destas pragas pode ser químico ou biológico, sendo que todas as técnicas empregadas no MIP são validadas por pesquisas e experiências de campo (CARVALHO; BARCELLOS, 2012), sendo que o controle químico preconizado consiste em duas aplicações espaçadas de 15 a 20 dias usando óleo mineral diluído a $1 \%$ com $50 \%$ da dose recomendada, de um inseticida fosforado.

\section{CONCLUSÕES}

Os resultados observados permitem concluir que durante todo período de observação, independente da temperatura e da umidade, ocorreu baixa incidência da cochonilha escama farinha. Apenas os meses de Janeiro e Julho de 2017/2018 se destacaram apresentando índice elevado de cochonilha. A umidade não interferiu nesse aumento, pois em Julho não houve precipitação, ao contrário do que observado em Janeiro quando ocorreram os maiores índices pluviométricos. 


\section{REFERÊNCIAS}

CARVALHO, N. L.; BARCELLOS, A. L. Adoção do manejo integrado de pragas baseado na percepção e educação ambiental. Revista Eletrônica em Gestão, Educação e Tecnologia Ambiental, Santa Maria, v. 5, n.5, p.749-766, 2012.

CORSEUIL, E. Combate às pragas da fruticultura. Porto Alegre: Secção de Informações e Propaganda Agrícola, 1958. 11p.

CORREIA, A. Fungos associados a Parlatoria cineria, em citros. Jaboticabal: SP, Universidade Estadual Paulista Julio Mesquista Filho, p.45, 1996.

FUNDECITRUS, Fundo de Defesa da Citricultura Pragas e Doenças - cochonilha escama farinha. Araraquara - SP, 2016.

Disponível:http://www.fundecitrus.com.br/doencas/escama-farinha/20. Visualizado em 06 de julho de 2019.

GARCÍA MORALES M.; DENNO B. D.; MILLER D. R.; MILLER G. L.; BEN-DOV Y.; HARDY N. B. ScaleNet: A literature-based model of scale insect biology and systematics. Database. p.7, 2018.

GOOGLE MAPS, Disponivel em:

<https://www.google.com.br/maps/place/Santa+F\%C3\%A9+do+Sul,+SP,+15775-

000/@-20.2885351,-

$50.9685484,2450 \mathrm{~m} /$ data $=! 3 \mathrm{~m} 1 ! 1 \mathrm{e} 3 ! 4 \mathrm{~m} 5 ! 3 \mathrm{~m} 4$ ! $1 \mathrm{~s} 0 \times 9499 \mathrm{c} 14 \mathrm{~d} 8 \mathrm{e} 677679: 0 \times c a 2041764$

52cd67d!8m2!3d-20.2114344!4d-50.9271748>. Acesso: Maio, 2019.

GRAVENA, S. Cochonilha branca: descontrolada em 2001. Manual Fitossanitário. Cordeirópolis: SP, v.24, n.1, p.71-82, 2003.

GRAVENA, S. História do controle de pragas na citricultura brasileira. Citrus Research \& Technology, Cordeirópolis, v.32, n.2, p.85-92, 2001.

GRAVENA, S., YAMAMOTO, P. T. Cochonilhas dos citros: principais espécies e seus inimigos naturais. São Paulo: Cibageigy, 1991. $32 \mathrm{p}$.

MUHAMmad, A.; PERVEEN, R.; NAQVI, A. U. N.; AHMED, K.; RAZA, G.; HUSSAIN, I. The Tribe Scymnini (Coccinellidae: Coleoptera) From Sindh Province, Pakistan. Journal of Insect Science, v. 15, n. 1. 2015.

OLIVEIRA, C. T.; ESTEVES FILHO, A. B.; PRADO, DIEGO L. D.; VALE, L. S. R. Levantamento Populacional De Artrópodes Em Tronco De Plantas Cítricas. Vale Do São Patrício: GO, V Congresso Estadual de Iniciação Científica e Tecnológica do IF Goiano IF Goiano- Campus Iporá 21 a 23 de setembro de 2016.

PERONTI, A. L. B. G.; MARTINELLI, N. M.; ALEXANDRINO, J. G.; JÚNIOR, A. L. M.; PENTEADO-DIAS, A. M.; ALMEIDA, L. M. Natural enemies associated with Maconellicoccus hirsutus (Hemiptera: Pseudococcidae) in the state of São Paulo, Brazil. Florida Entomologist, v. 99, p. 21-25, 2016. 
TAVARES, A.; Prevalência de Fungos associados a Parlatoria ziziphi. Jaboticabal: SP, Universidade Estadual Paulista Julio Mesquista Filho, p.45, 1997.

WOLFF, V. R. S.; PULZ, C. E.; SILVA, D. C.; MEZZOMO, J. B.; PRADE, C. A. Inimigos naturais associados à Diaspididae (Hemiptera, Sternorrhyncha), ocorrentes em Citrus sinensis (Linnaeus) Osbeck, no Rio Grande do Sul, Brasil: I- Joaninhas e fungos entomopatogênicos. Arquivos do Instituto Biológico, São Paulo, n. 71, v. 3, p. 355-361, 2004.

YAMAMOTO, P. T.; PAIVA, P. E. B. Evolução e manejo dos insetos sugadores dos citros. Jaboticabal: SP, UNESP, p.265, 2014. 